\title{
Indirect determination of the mechanical properties of stochastic lattices
}

\author{
Georgios Maliaris ${ }^{1, *}$, Theologos Lazaridis ${ }^{2}$, Ilias T. Sarafis ${ }^{2}$ and Sofia Kavafaki $^{2}$ \\ ${ }^{1}$ Mechatronics \& Systems Automation Laboratory, Department of Electrical \& Computer \\ Engineering, School of Engineering, Democritus University of Thrace, GR67100, Xanthi, Greece \\ ${ }^{2}$ Mechanical Engineering Department, Technological Educational Institute of East Macedonia- \\ Thrace, Agios Loukas, GR65404, Kavala, Greece
}

\begin{abstract}
Determination of the mechanical behaviour of lattice structures has become a necessity to successfully implement lightweight concepts in various applications. Due to the large surface to volume ratio of these structures, the computational effort required for the FE simulation of models incorporating lattices is significantly increased mainly because of the large finite elements number which are necessary to accurately describe the complex input geometry. In this work a simple solution is employed in order to calculate the stress - strain properties, using a bilinear material law and equivalent bulk geometry. The verification of the FE model is fulfilled through the comparison of the lattice deformation and compressive force between experimental data and FE calculated results. After the verification of the FE model, it was possible to determine the mechanical behaviour of stochastic lattices, for an extended range of the investigated parameters, using only computational tools.
\end{abstract}

\section{Introduction}

Weight reduction of structures, through the implementation of 3D lattices, has become a necessity in many industries such as aerospace and automotive. These structures are also characterised by their unique properties such as high strength to weight ratios as well as energy absorption characteristics [1]. Nowadays, metal foams and honeycomb structures are used for a broad range of applications in aerospace/ automotive industries and recently in orthopaedics and they are manufactured using various production methods [2]. Two different cellular arrangements are commonly introduced; ordered and stochastic one. The geometry of random lattices (metal foams) consists of irregular, non-periodic cells which form a network of interconnecting walls or struts. On the other hand, regular lattice structures (honeycomb) are formed by the repetitive placement, at the same distance in 3D space, of a representative cell.

Understanding mechanical behaviour is the key parameter for the proper adoption of cellular materials to various applications. Experimental testing combined with finite element analysis has proved to be a solid method for material characterization. In the case of cellular geometries with stochastic arrangement, experimental testing is an easy task in

* Corresponding author: gmaliari@ee.duth.gr 
contrast to FEA where geometry definition, an essential step towards seamlessly FE model setup, imposes difficulties due to random structure nature. Initial efforts for foam modelling relied on previously submitted articles on foam froth geometry approximation. In these investigations the Kelvin cell was used which partitions space with minimum surface area for identical cells $[3,4,5]$. A promising method, recently employed by many researchers, is the generation of irregular cells applying the Voronoi tessellation technique. This technic is used to partition 3D space into random polyhedra. The geometric features of the generated polyhedra are later used in lattice structure modelling.

The mechanical behaviour of cellular solids has been thoroughly investigated by various researchers the last 15 years. The elastic response of open cell foams was studied by [6, 7 , 8]. In their investigations researchers considered the effect of various parameters such as relative density, cell irregularity, cell shape and strut cross sectional variations. It was found that relative density and cell irregularity are the dominant parameters affecting the elastic moduli. Also, as energy absorbers, foams are frequently subjected to dynamic loadings or impacts. Therefore, it is quite significant to analyse the large plastic deformation behaviour considering also dynamic phenomena such as rate dependent material properties. Large plastic deformation regime of cellular materials consists of two regions; extended stress plateau and densification region [2]. In order to capture the densification effect through FE analysis, self-contact between struts should be implemented in the developed FE models. The later requirement increases the computation effort by a large margin, so simplification of the strut FE modelling is a necessary step towards efficient computational time management. Two dimensional FE models $[9,10]$ and beam-based strut realization $[6,10$, $11]$ are the main methods which are commonly applied.

The geometrical characterization of stochastic cellular structures is achieved through the determination of geometric parameters such as cell irregularity, strut cross sectional shape, strut equivalent radius, porosity. Up to recently it was not possible to prescribe these parameters, since they are coupled to the applied processing method, thus they follow physical laws and remain uncontrolled parameters. The last years, the emergence of various additive manufacturing methods, with enhanced accuracy, rendered feasible the materialization of stochastic and ordered lattice structures. The mechanical behaviour of 3D - printed ordinate structures using the DMLS (Direct Metal Laser Sintering) or SLM (Selective Laser Melting) additive technologies where investigated by several researchers [12 - 14]. The failure mechanisms of additively manufactured porous biomaterials considering porosity and unit cell type was investigated in [13]. The effects of build orientation and heat treatment on the microstructure and mechanical properties of selective laser melted Ti6Al4V lattice structures was explored in [14]. The above mentioned research papers were focused on metallic lattice structures which were modelled using specific unit cells.

In this work, a Voronoi tessellation based algorithm is employed, which has been developed for modelling stochastic lattice structures [15]. With the proposed algorithm, is possible to generate CAD geometry with controllable structural parameters, such as porosity, cell number and strut thickness. The digital structures were transformed into physical objects through the combination of 3D printing technics and lost wax casting. Until now, the only way to materialise such structures into physical objects was feasible through additive manufacturing methods such as Selective Laser Sintering/ Melting (SLS/ SLM). Lost wax casting possesses numerous advantages against SLS or SLA, with the major one being the material variety. On the other hand, several trials are required to calibrate the process parameters to have successful castings, and also, the volume of the specimen is limited, which is the major drawback of the specific casting process.

\section{Materials and methods}




\subsection{Solid modeling}

The Voronoi tessellation (or Voronoi diagram) is a space partitioning method which creates polyhedra, when applied to a 3D geometry (a control volume) that share a common characteristic. The distance between the seed point of a polyhedron and any point included in the specific polyhedron is the minimum one with respect to other seed points. The tessellation is applied to $\mathrm{N}$ randomly distributed seed points into a control volume, which is usually a simple geometry such as cube, cylinder, sphere, etc.. The employed seed point distribution method is of a great interest since it defines the statistical distribution of pivotal geometric characteristics. In the next step, the neighbouring points are connected with lines which are then bisected by their perpendicular planes. For a given seed point, its Voronoi cell is defined by the smallest polyhedron that contains that point. The above mentioned procedure is presented in figure 1 for 10 seed points.

Random point distribution Vertical planes positioning

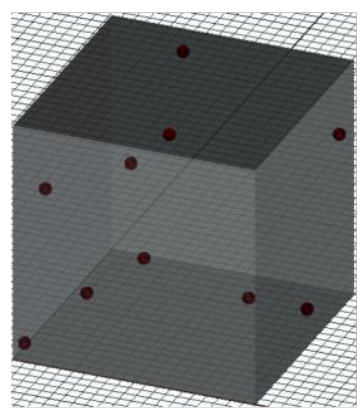

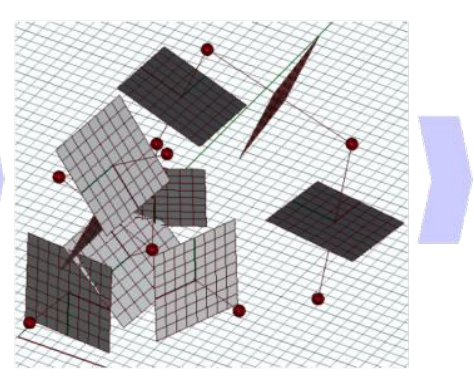

Voronoi cells generation

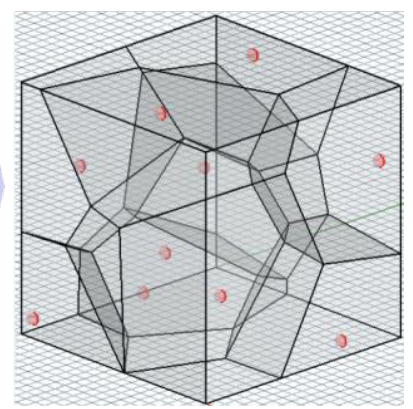

Fig 1: The Voronoi tessellation procedure

The edges of the Voronoi cells form the skeleton which can be appropriately dressed to represent the struts of the lattice structure. Most of the researchers use the vertices of the cells in order to define beam elements during the FE model definition, as it can be observed in figure $2[4,6,10,16,17,18]$.
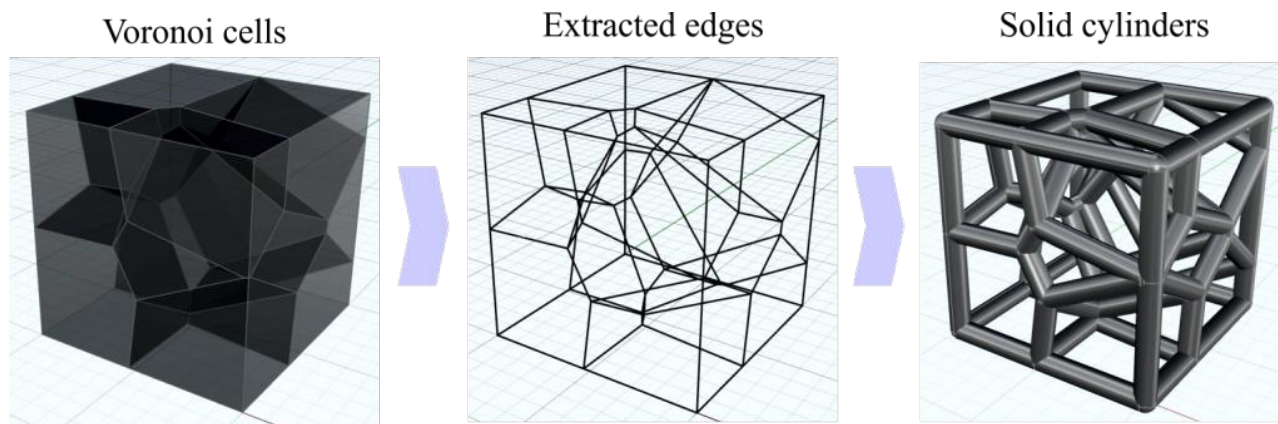

Fig 2: Solid modelling procedure

Although this simplification leads to geometries that do not capture the cross section variation along strut length and mass concentration at the junctions, is a preferred method of strut modelling in order to limit the required computational effort. Seven digital structures where modelled (see table 1), 4 with the same number of cells $(125,5$ per axis) but with different porosities ( $\sim 62, \sim 71, \sim 79$ and $\sim 87 \%$ ), as it can be observed in figure 3 . 

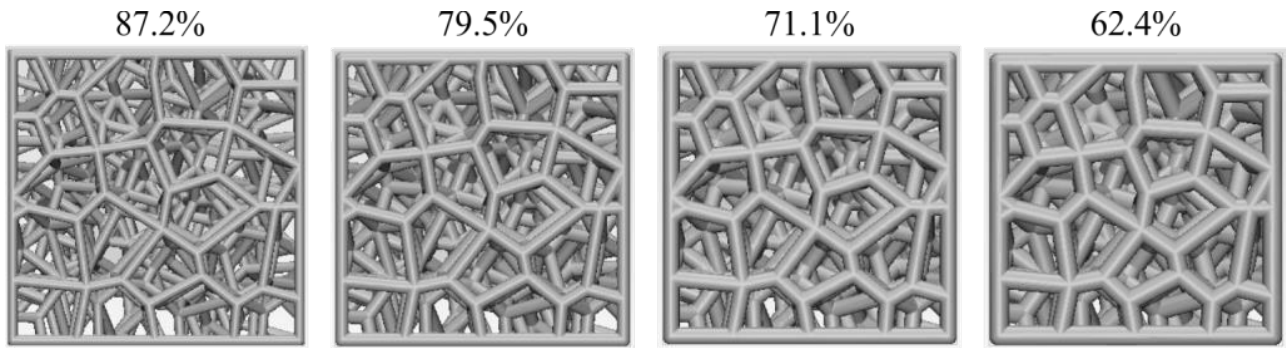

Fig 3: Modelled stochastic lattices with different porosities

Another two lattices were manufactured with the same porosity ( $79 \%)$ but with different number of cells $(100, \& 150)$ and strut thicknesses and one structure with porosity of $\sim 79 \%$, 125 cells but with a different distribution of the seed points used by the Voronoi tessellation algorithm. All stochastic lattices had the same external dimensions (40X40X40mm). Three copies each solid model were manufactured using a castable photosensitive resin (Bluecast LS - www.bluecast.info) and a high resolution SLA 3D printer (Formlabs FORM2). The layer thickness was set equal to $50 \mu \mathrm{m}$. After printing the samples were UV post-cured for 30 minutes. Although the external dimensions of the specimens were accurate enough, deviations were observed during the conduction of strut diameter measurements. As it was concluded, the struts had become larger by a percentage of approximately $5-10 \%$.

Table 1. Design parameters of modelled stochastic lattices

\begin{tabular}{|c|c|c|c|c|}
\hline A/A & Number of cells & Strut radius [mm] & Porosity [\%] & Random seed number \\
\hline 1 & 125 & 0.75 & 87.2 & 53 \\
\hline 2 & 125 & 1.00 & 79.2 & 53 \\
\hline 3 & 100 & 1.05 & 79.2 & 53 \\
\hline 4 & 125 & 1.00 & 79.2 & 87 \\
\hline 5 & 150 & 0.95 & 79.2 & 53 \\
\hline 6 & 125 & 1.25 & 71.1 & 53 \\
\hline 7 & 125 & 1.50 & 62.4 & 53 \\
\hline
\end{tabular}

\subsection{Lattice structure manufacturing and mechanical testing}

An A356 aluminium alloy (SAG GmbH) was used to cast the stochastic lattices (SpeedCast $220 \mathrm{MJ}$ - Orotig S.r.L). The specific machine has automated casting cycles, where the metal is melted by the electric arc in presence of Argon-gas. When the casting takes place, the molten metal is pushed down into the flask by a controlled Argon-gas overpressure. This makes possible to obtain very compact, oxidation-free casting extremely accurate in details and quite precise and fitting even in very diminutive sections, thus is the perfect candidate for manufacturing the stochastic lattices. Manufactured samples were subjected to uniaxial compression tests (INSTRON 8801 UTM - Instron), where their mechanical response was registered in the form of compressive load - displacement curves. All specimens were slightly prestressed $(<10 \mathrm{~N})$ in order to eliminate any planarity errors. Thereafter, a constant compression displacement rate was applied to the specimen, equal to $2 \mathrm{~mm} / \mathrm{min}$. 


\section{Results}

\subsection{Experimental results}

After casting and removal of the investment material it was clear that the quality of the specimens' surface suffered from pores and cracks, especially the specimens having small strut diameter (see figure 4). The findings were attributed to the incompatibility of the investment material with the photocurable resin and also it seems that the provided resin burn out schedule was not fully calibrated to the specific resin type, causing the presence of residue during casting process. The specific defects were expected to have an impact on the mechanical behaviour of the lattice structures. Although the surface quality was compromised, the dimensions of the produced specimens where very accurate, indicating that the aluminium's shrinkage compensated the enlarged struts' diameter.
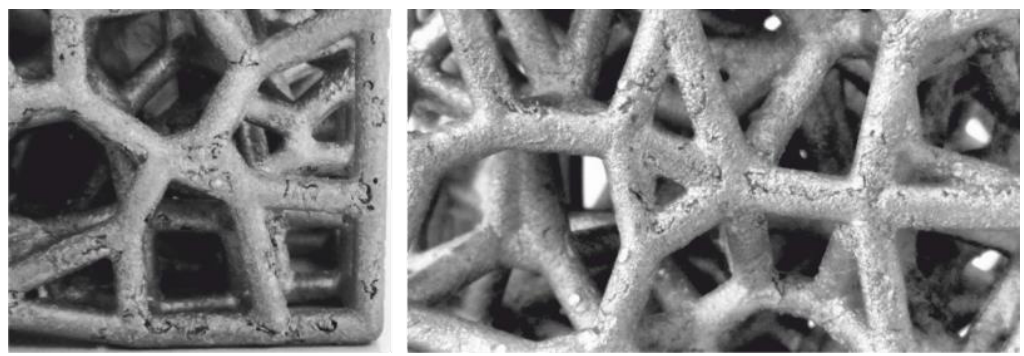

Fig 4: Defects on the surface of as cast specimens

The load- displacement curves for all specimens are depicted in figure 5. As expected, the experimental results reveal a strong relation between porosity and resulting compression load. On the other hand, lattices with the same porosity of $79.5 \%$ (specimens $2-5$, see table 1) exhibit almost the same reaction force. Two of these lattices have different number of cells $(100 \& 150$ - specimens 3,5$)$ with appropriate radii to keep the porosity constant. Also, one more lattice structure (specimen 4) has a different random seed number distribution, keeping the same radius value. None of these variations showed to have a significant effect on the compression force.

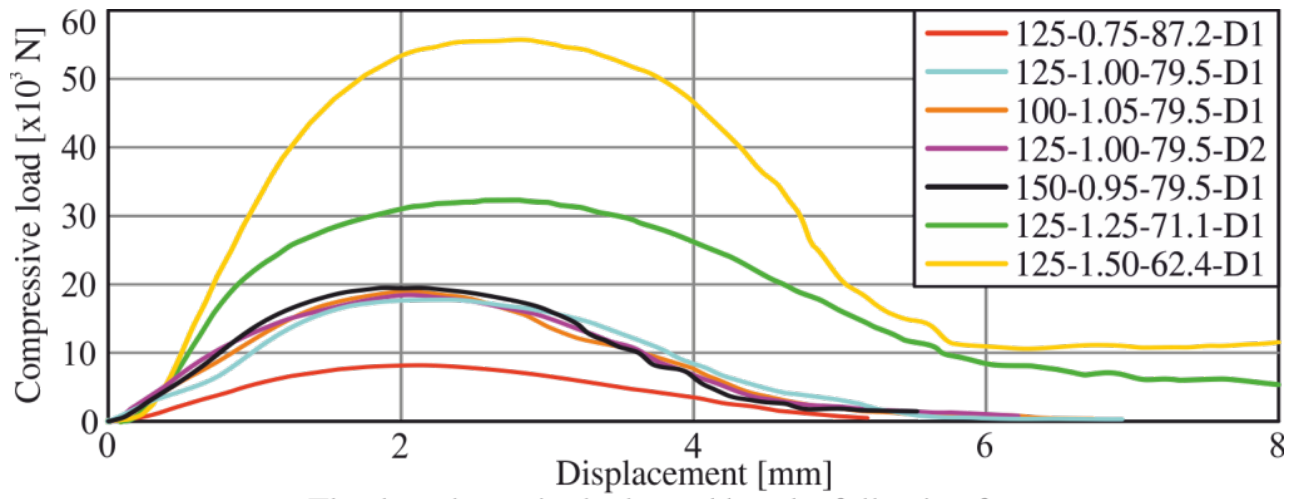

The data shown in the legend has the following format:

Number of cells-Strut radius-Porosity-Seed points distribution

Fig 5: Load - displacement curves derived from the compression tests 


\subsection{FE assisted calculations}

The scope of this work is to provide a simple mean for the determination of the mechanical behaviour of stochastic lattice structures. Within this frame, the stress-strain properties of an equivalent bulk geometry, with identical external dimensions to the modelled lattice structures, were determined in a stochastic approach by continuously altering the non-linear material law of the bulk geometry until achieving an identical compressive response. A bilinear kinematic hardening law was selected among the available non linear material models, to restrict the number of the variables that had to be determined. The optimum mesh density was determined based on a convergence study, this was achieved through consecutive simulations of a reference displacement $(2 \mathrm{~mm})$, while altering the mesh density. Once two consecutive simulations result in a deviation of the monitored reaction force values of $0.2 \%$ [19], the grid was considered as 'mesh independent' and accepted for all further simulations. The final mesh consisted of 12.608 solid elements, with mean element side length of $2 \mathrm{~mm}$. The displacement of the top plate as restricted to $2 \mathrm{~mm}$, since the reaction force is getting its maximum value between $2-3 \mathrm{~mm}$ of compression which in turn equals to a strain value to $5-7.5 \%$. Determining the material properties through FE simulation of the compression process for specimen 7 , resulted to the force - displacement curve depicted in figure 6.

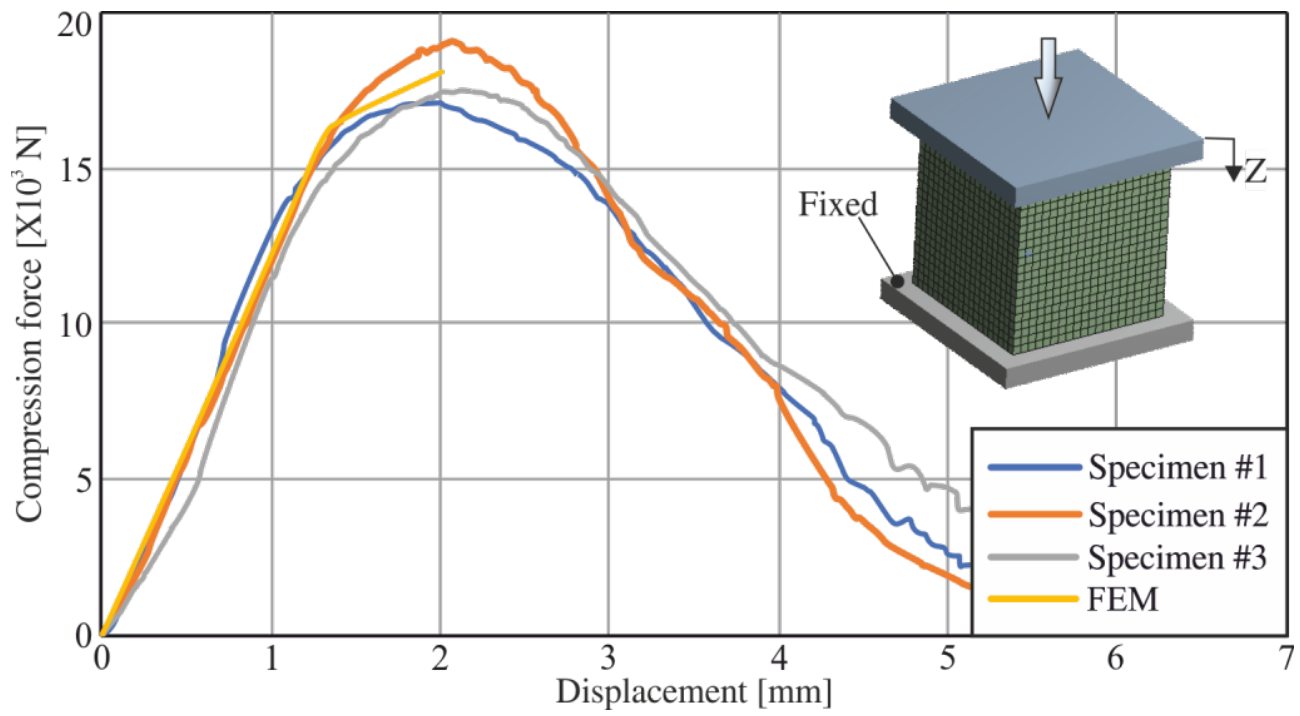

Fig 6: Load - displacement curves derived from the compression tests as well as the FE simulation

\section{Discussion}

One of the characteristic properties of metallic foams and lattice structures is the formation of a plateau which is extended for a wide strain range [2]. The load value which corresponds to the plateau level and the deformation range in which the force remains constant is a metric of the energy absorption capability of a specific foam or lattice structure. In this work, as it can be seen in figure 5, no plateau region is formed. As it was mentioned, the surface quality of the as cast specimens was impaired by the formation of crack and pores. Although it was not obvious initially, during compression testing it was more than clear that material integrity was also affected. At early displacement levels (1$2 \mathrm{~mm}$ ), the struts did not deform, but rather started to brake. When displacement exceeded $3 \mathrm{~mm}$, most of the lattice structures were heavily damaged with a large number of disjoint 
struts been present. As it was concluded, A356 alloy, as well as high -Si aluminum alloys, become brittle during casting and fail under load for strains up to $5 \%$, which is in agreement with the experimental observations. In these cases, the material can become malleable and permits elongation up to $20-30 \%$ when appropriately heat treated.

Due to this issue, only the first half of the force - displacement curves were considered for material characterization and especially the portion from the beginning of the experiment up to the point were force reaches its maximum value. The results of the FE simulations for lattices with porosity 87.2 and $79.2 \%$ are presented in figure 7.

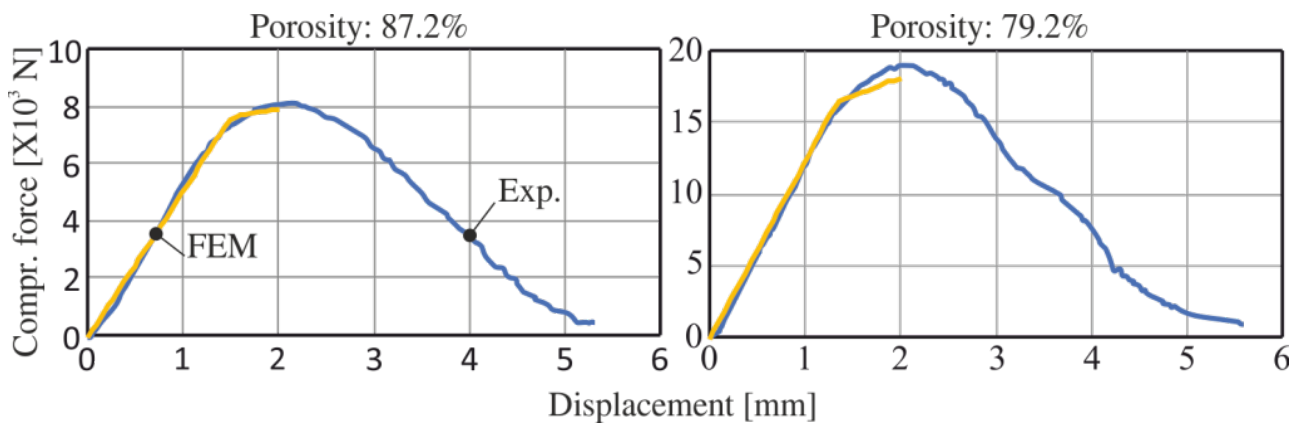

Fig 7:Experimental and FE calculated force - displacement curves for stochastic lattice with porosities of 87.2 and $79.2 \%$

Bilinear kinematic hardening model is described by defining young modulus, yield stress and the tangent modulus for the second line of the bilinear model. Initially the young modulus is calculated so as the slope of the calculated force - displacement curve to match the experimental one. Then the yield stress and the tangent modulus are calibrated by comparing the two curves. This procedure was applied for specimens 1, 2, 6, 7. In figure 8, the calculated values for the aforementioned variables, as well as the reaction (compression) force, are plotted against porosity.
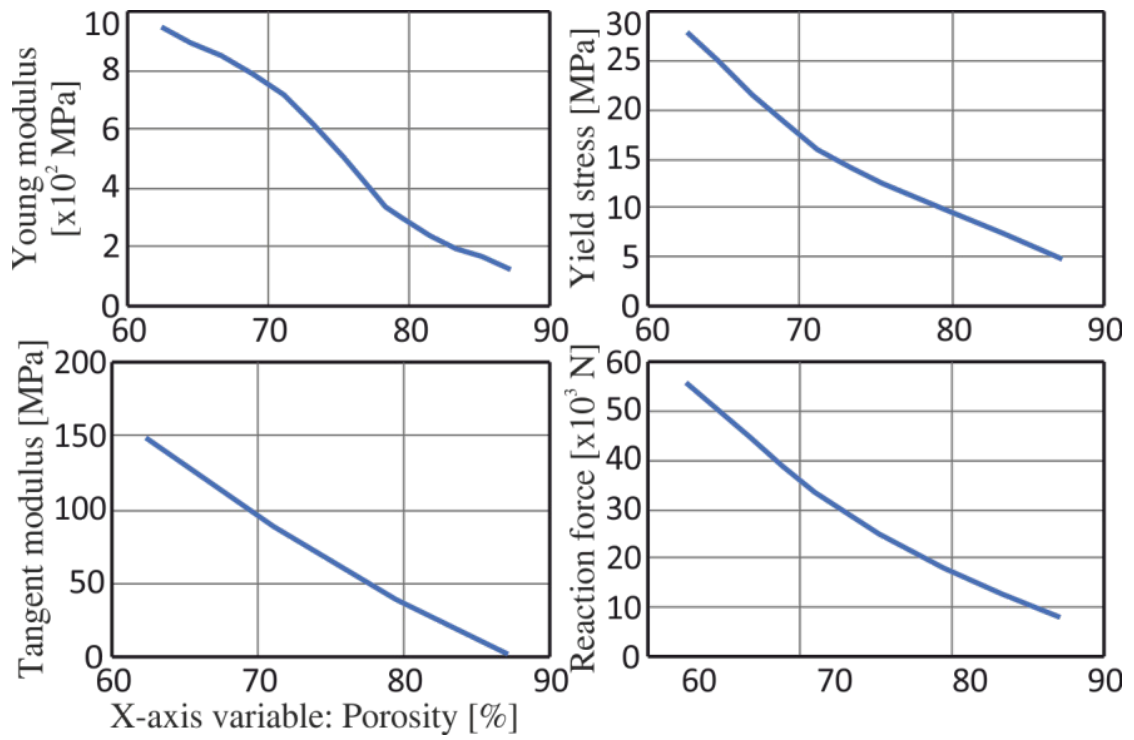

Fig 8: Relations between porosity and calculated variables

As it can be observed there is an almost linear relation between the calculated variables of the bilinear material model and porosity, rendering feasible the determination of simple mathematical functions, which are presented in the next table. 
Table 2. Determination of mathematical functions

\begin{tabular}{|l|l|}
\hline Variable & Function \\
\hline Young modulus [MPa] & $y=-34.826 x+3138.7$ \\
\hline Yield stress [MPa] & $y=-0.9099 x+83.04$ \\
\hline Tangent modulus [MPa] & $y=-5.9424 x+516.73$ \\
\hline Reaction force [kN] & $y=-1921.7 x+173205$ \\
\hline
\end{tabular}

The specific functions can assist with the calculation of the reaction force, if the porosity is known, or in reverse, what the porosity should be in order to achieve a specific maximum reaction force value. After the calculation of the appropriate porosity value, the rest variables which determine the bilinear material model can be also calculated. The calculated variables are valid for small material elongations, up to 5\%.

\section{Conclusions}

The determination of the mechanical properties of random lattices was succeeded, in the form of linear equations, with the aid of algorithmic 3D modeling, 3D printing, investment casting and FE assisted calculations. With the specific procedure it is possible to predetermine the mechanical behavior of stochastic lattices, especially in the elastic strain regime, rendering feasible the minimization of the necessary computational effort.

\section{References}

1. J. Banhart, JOM 52, 22-27 [2000]

2. L. J. Gibson, M. F. Ashby, Cellular Solids - Structure and Properties, Cambridge University Press, Cambridge [1997]

3. K. Li, X. L. Gao, G. Subhash, J. Mech. Phys. Solids 54, 783-806 [2006]

4. M. Alkhader, M. Vural, Int. J. Eng. Sci. 46, 1035-1051 [2008]

5. S. V. Raj, Mat. Sci. Eng. A-Struct. 528, 5289-5295 [2011]

6. W. Y. Jang, S. Kyriakides, A. M. Kraynik, Int. J. of Solids Struct. 47, 2872-2883 [2010]

7. L. Gong, S. Kyriakides, Int. J. of Solids Struct. 42, 1381-1399 [2005]

8. W. Y. Jang, A. M. Kraynik, S. Kyriakides, Int. J. of Solids Struct. 45, 1845-1875 [2008]

9. M. Borovinšek, Z. Ren, Materialwiss. Werkst. 39, 114-120 [2008]

10. J. Alsayednoor, P. Harrison, Z. Guo, Mech. Mater. 66, 7-20 [2013]

11. N. Michailidis, Mat. Sci. Eng. A-Struct. 528, 4204-4208 [2011]

12. S. Ahmadi, S. Yavari, R. Wauthle, B. Pouran, J. Schrooten, H. Weinans, A. Zadpoor, Materials 8, 1871-1896 [2015]

13. J. Kadkhodapour, H. Montazerian, A. C. Darabi, A. P. Anaraki, S. M. Ahmadi, A. Zadpoor, S. Schmauder, J Mech Behav Biomed Mater 50, 180-191 [2015]

14. R. Wauthle, B. Vrancken, B. Beynaerts, K. Jorissen, J. Schrooten, J. P. Kruth, J. Van Humbeeck, Addit. Manuf. 5, 77-84 [2015]

15. G. Maliaris, I. Sarafis, Solid State Phenom. 258, 225-228 [2017]

16. S. Gaitanaros, S. Kyriakides, Int. J. Impact Eng. 82, 3-13 [2015]

17. S. Gaitanaros, S. Kyriakides, A. M. Kraynik, Int. J. of Solids Struct. 49, 2733-2743 [2012]

18. O. E. Sotomayor, H. V. Tippur, Acta Mater. 78, 301-313 [2014]

19. O.C. Zienkiewicz, R.L. Taylor, The finite element method, New York (NY): McGrawHill. [1989] 